\title{
Primary central nervous malignant melanoma in the bathing trunk naevus syndrome
}

\author{
J.R. Salisbury and P.E. Rose \\ Department of Morbid Anatomy, King's College School of Medicine and Dentistry, London and Hurstwood Park \\ Neurological Centre, West Sussex, UK.
}

\begin{abstract}
Summary: The gross and microscopic features of an unusual case of neurocutaneous melanosis are described. This is an example of the rare association between a primary central nervous system malignant melanoma and a giant congenital melanocytic naevus of 'bathing trunk' distribution.
\end{abstract}

\section{Introduction}

Cases of cutaneous melanocytic pigmentation with accompanying leptomeningeal melanocytosis are often referred to as neurocutaneous melanosis. ${ }^{1,2}$ In some, but not all, instances the leptomeningeal melanocytes undergo malignant change ${ }^{1,3,4}$ although the histological features of malignancy are often lacking. Fox et al. ${ }^{1}$ reported one such case with unequivocal melanoma and reviewed 12 previous cases. Reed et al. described a further case and reviewed 3 more from the older literature. ${ }^{3}$ Four other cases have been described since., ${ }^{2,5}$ The rarity of this association between primary meningeal malignant melanoma and giant congenital melanocytic naevi prompts us to describe a further example.

\section{Case report}

The patient was born the second of twins, the first twin being normal. At birth she had a large pigmented naevus covering the bathing trunk area and extending down to the knees. Small round pigmented naevi were scattered over the rest of the skin surface. She remained well until age 13 when she developed complex partial and generalized epileptic seizures which were well controlled with carbamazepine. At age 26 she was admitted to hospital with a 3-week history of headache and nausea and on examination was clearly ill but without signs of meningism. Microscopic examination of cerebrospinal fluid showed atypical cells which, at that time, were interpreted as being epithelial. Computer-assisted tomographic and

Correspondence: J.R. Salisbury, B.Sc., M.B., M.R.C.Path., Department of Morbid Anatomy, King's College School of Medicine and Dentistry, Bessemer Road, London SE5 9PJ, UK.

Accepted: 21 December 1988 nuclear magnetic resonance scans demonstrated numerous lesions over both cerebral hemispheres. She was treated with high doses of dexamethasone and given cranial irradiation, which brought about some improvement, but she then developed visual impairment with central scotomata and psychotic symptoms. Her health gradually deteriorated and she died 5 months later.

\section{Autopsy findings}

The main bathing trunk naevus and several smaller naevi were sampled for microscopic examination and showed benign intradermal melanocytes with no junctional activity. The extensive nature of the naevi precluded examination of them all. The thoracic and abdominal organs were normal on both gross and microscopic examination, specifically the pituitary, thyroid and adrenal glands were all normal.

The brain weighed $1311 \mathrm{~g}$. Numerous pigmented lesions, 1 to $5 \mathrm{~mm}$ in diameter, were present over the cerebral hemispheres. On the inferior left temporal surface the lesions formed a contiguous plaque, measuring $4 \mathrm{~cm}$ anteroposteriorly and $3 \mathrm{~cm}$ laterally, which involved the parahippocampal gyrus.

Coronal slices confirmed the presence of hundreds of intracortical melanomata in all regions of the cortex and in the basal ganglia. In the left inferior temporal cortex the tumour deposits formed a continuous sheet throughout the surface (Figure 1). This was of particular interest, in view of the clinical history, as were the presence of bilateral amygdaloidal and large thalamic deposits (Figure 1). The cerebellum, medulla oblongata and spinal cord appeared macroscopically normal.

Microscopic examination revealed the presence of a nearly contiguous malignant melanomatous infiltrate,

(C) The Fellowship of Postgraduate Medicine, 1989 


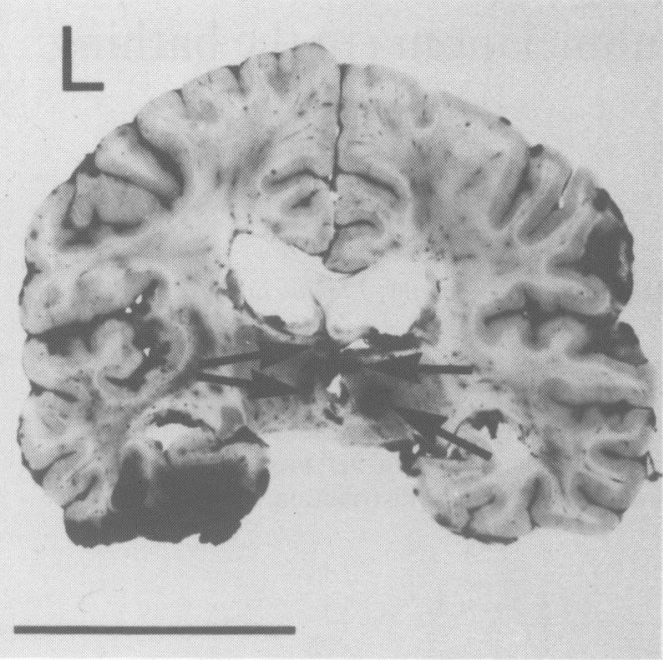

Figure 1 Coronal slice of the fixed brain at the level of the lateral geniculate bodies. Note multiple generally medially situated thalamic tumours (arrows) and contiguous cortical infiltration in the left inferior temporal cortex. Bar $=50 \mathrm{~mm}$.

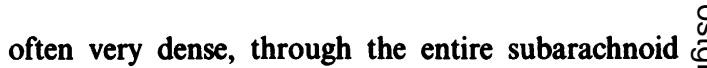
space. In both the cerebral cortex and the basal ganglia there were numerous, generally densely pigmented, foci of neoplastic invasion in the Virchow-Robin $\mathbb{D}$ spaces and grey matter. In the cord and nerve roots, $c$ invasion of the perivascular spaces was sparse and $\ddot{\Rightarrow}$ there was little direct invasion of the spinal cord or $\stackrel{\oplus}{\stackrel{9}{+}}$ cranial and peripheral root parenchyma although $\bar{c}$ melanoma had surrounded and invaded the optic nerves. There was no evidence of pre-existing benign central nervous system melanocytic or neuroglial hamartomas.

Cytologically the tumour cells were round, exhibited both cellular and nuclear pleomorphism and ranged from 10 to $50 \mu \mathrm{m}$ across. They were generally $\vec{\omega}$ non-pigmented; only occasional cells containing a few coarse melanin granules (Figure 2).

\section{Discussion}

The association of primary meningeal malignant melanoma with giant congenital naevi of the skin is $\dot{\omega}$ rare. Giant congenital melanocytic naevi have been

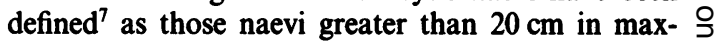
imum diameter. They frequently have a 'garment-

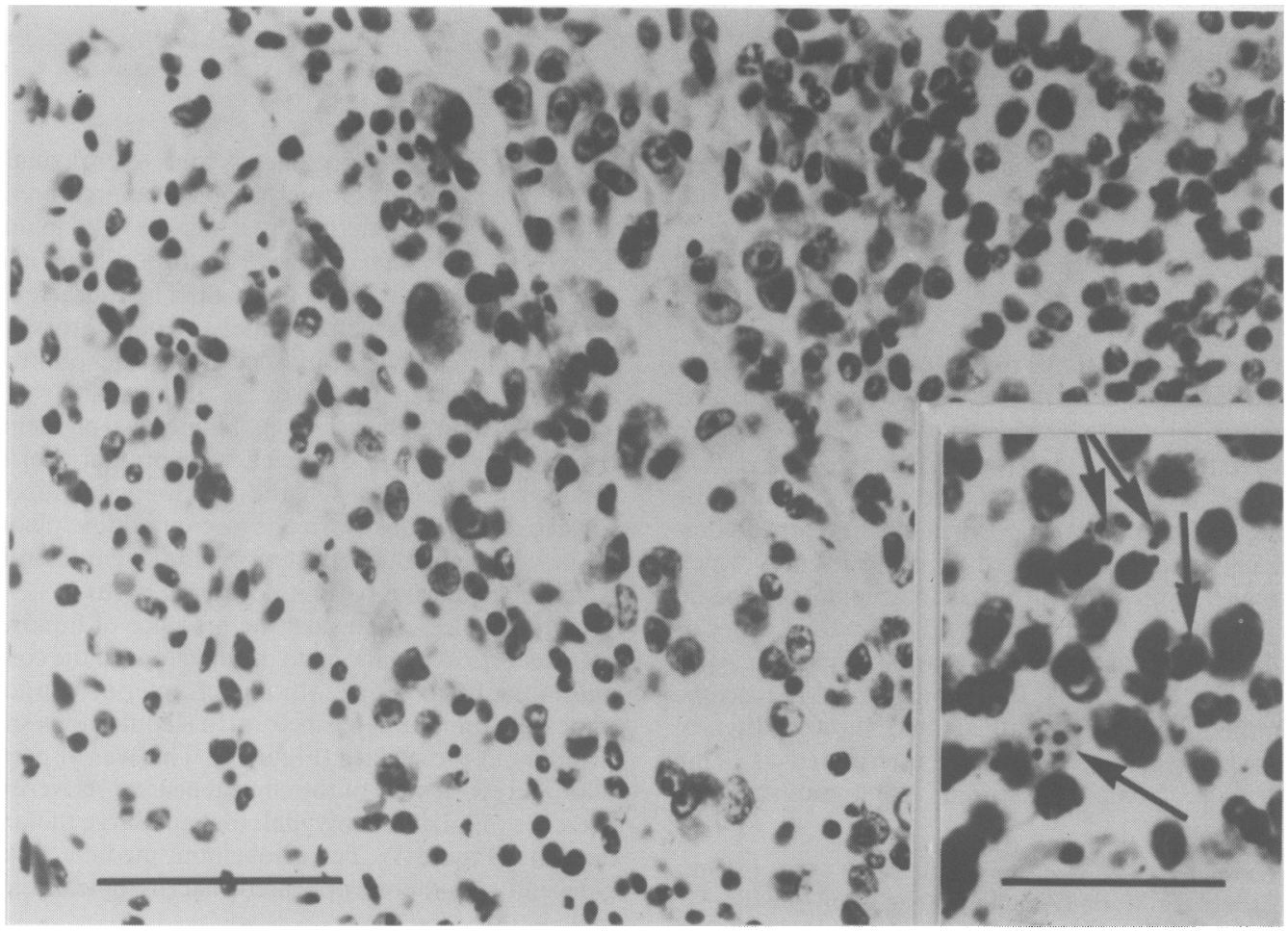

Figure 2 Leptomeningeal tumour cells showing both nuclear and cellular pleomorphism. Bar $=50 \mu \mathrm{m}$. Inset: Occasional cells contain a few coarse melanin granules (arrowed). Bar $=100 \mu \mathrm{m}$. 
type' distribution. Malignant melanomas arise in about $12 \%$ of these giant naevi, ${ }^{7}$ usually within the intradermal part of the naevus rather than at the dermo-epidermal junction. ${ }^{3,8}$ In the absence of cutaneous melanoma it is almost certain that the malignant melanomatosis in the present case arose in the central nervous system. Intracranial malignant melanomas have been reported in association with a naevus of Ota ${ }^{9}$ and with a cellular blue naevus ${ }^{10}$ as well as with giant congenital melanocytic naevi. ${ }^{1-6}$

The present case is unusual in the comparatively late ages at which the epilepsy commenced, and at which the neurological melanomatosis became manifest; evidence of central nervous system disease presents in the majority of cases of neurocutaneous melanosis during childhood, and the disease pursues a generally more rapid course. Although the exact nature of the agents which precipitated the epilepsy in our case must remain speculative, it is difficult to account for the abrupt onset of epilepsy at the age of 13 years except on the basis of an underlying slowly progressive

\section{References}

1. Fox, H., Emery, J.L., Goodbody, R.A. \& Yates, P.O. Neuro-cutaneous melanosis. Arch Dis Child 1964, 39: 508-516.

2. Slaughter, J.C., Hardman, J.M., Kempe, L.G. \& Earle, K.M. Neurocutaneous melanosis and leptomeningeal melanomatosis in children. Arch Pathol 1969, 88: 298-304.

3. Reed, W.B., Becker, W.S. Sr., Becker,W.S. JR \& Nickel, W.R. Giant pigmented nevi, melanoma and leptomeningeal melanocytosis. Arch Dermatol 1965, 91: 100-119.

4. Touraine, A. Les melanoses neuro-cutanees. Ann Dermatol Syph 1949, : 489-524.

5. Williams, H.I. Primary malignant meningeal melanoma associated with benign hairy nevi. J Pathol 1969, 99: $171-172$.

6. Willis, R.A. The hamartomatous syndromes: Their clinical, pathological and fundamental aspects. Med $J$ Aust 1965, 1: 827-833. central nervous system melanosis which had by that age evolved to the extent of causing symptoms. The only other case we have discovered in which a period of several years elapsed between the onset of epilepsy and death is the second case of Slaughter et al. ${ }^{2}$ in which epilepsy commenced at 4 years of age and death occurred at 14 years. The visual impairment in our patient was probably largely due to the infiltration in and around the optic nerves, and possibly to intracortical deposits in the occipital cortices. The basal ganglia and temporal cortical deposits probably contributed in a large part to the generation of psychotic symptoms.

In conclusion, this case report describes an example of the rare association between congenital neurocutaneous melanosis with giant bathing trunk naevus and the subsequent development of a primary malignant melanoma of the central nervous system. Our case is exceptional with regard to the late onset of symptoms and death.

7. Kopf, A.W., Bart, R.S. \& Hennessey, P. Congenital nevocytic nevi and malignant melanomas. J Am Acad Dermatol 1979, 1: 123-130.

8. Penman, H.G. \& Stringer, H.C.W. Malignant transformation in giant congenital pigmented nevus. Arch Dermatol 1971, 103: 428-432.

9. Sagar, H.J., Ilgren, E.B. \& Adams, C.B.T. Nevus of Ota associated with meningeal melanosis and intracranial melanoma. Case report. J Neurosurg 1983, 58: 280-283.

10. Findler, G., Hoffman, H.J., Thomson, H.G. \& Becker, L. Giant nevus of the scalp associated with intracranial pigmentation. Case report. J Neurosurg 1981, 54: $108-112$. 